\title{
Molecular Dynamics Simulations of Nanolubricant Films of Alkali Glasses under Sliding Asperity Contacts
}

\author{
Thi D. Ta, A. Kiet Tieu*, Bach H. Tran
}

School of Mechanical, Materials Mechatronic and Biomedical Engineering, Faculty of Engineering and Information Sciences (EIS), University of Wollongong, Northfield Avenue, Wollongong, NSW, 2522, Australia

Corresponding author: Kiet Tieu

ktieu@uow.edu.au 

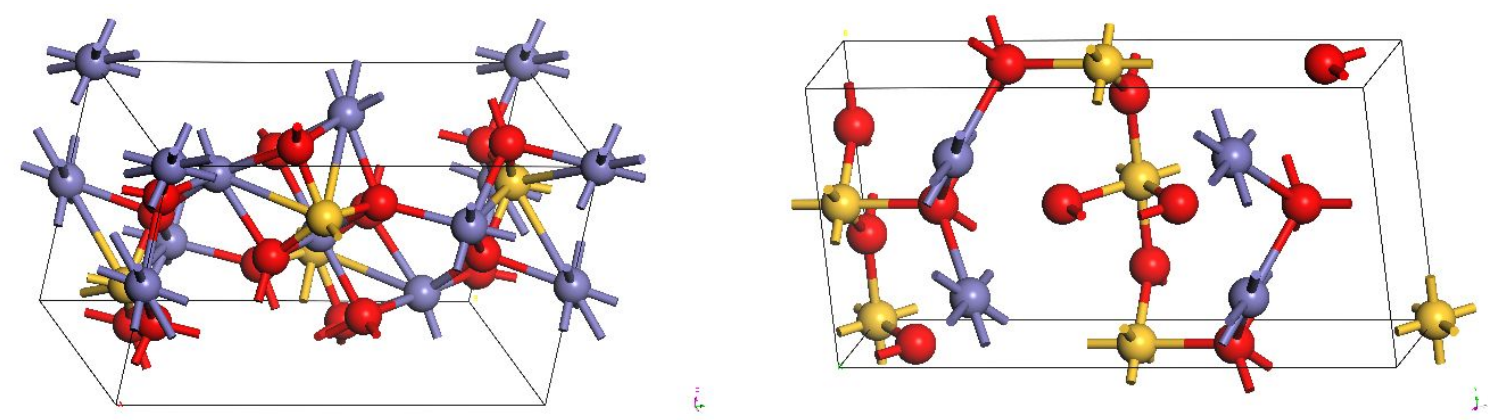

Fig. S1 Crystalline structures of $\mathrm{Fe}_{2} \mathrm{SiO}_{4}$ (left) and $\mathrm{FeSiO}_{3}$ (right). Purple, yellow, and red colours are corresponding for $\mathrm{Fe}, \mathrm{Si}$, and $\mathrm{O}$ elements.

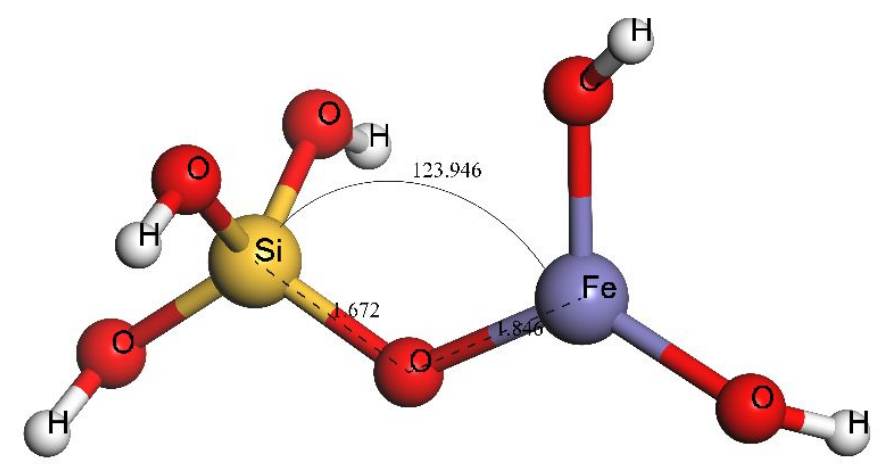

Fig. S2 Molecular structure of $\mathrm{Si}(\mathrm{HO})_{3}-\mathrm{O}-\mathrm{Fe}(\mathrm{OH})_{2}$ molecule. Purple, yellow, and red colours are corresponding for $\mathrm{Fe}, \mathrm{Si}$, and $\mathrm{O}$ elements.

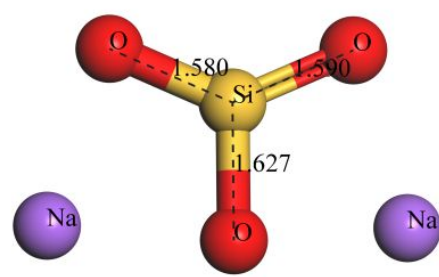

$\mathrm{Na}_{2} \mathrm{SiO}_{3}$ molecule

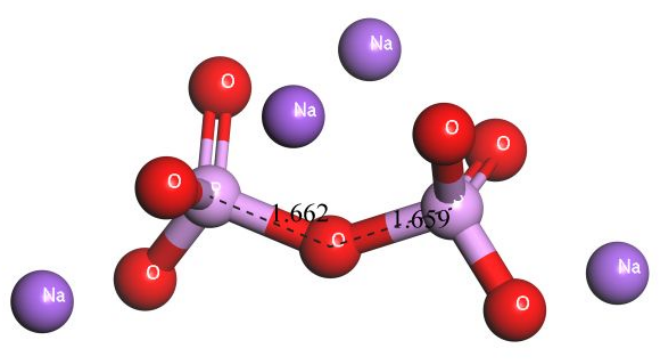

$\mathrm{Na}_{4} \mathrm{P}_{2} \mathrm{O}_{7}$ molecule

Fig. S3 Optimized molecular structure of isolated molecules of $\mathrm{Na}_{2} \mathrm{SiO}_{3}$ and $\mathrm{Na}_{4} \mathrm{P}_{2} \mathrm{O}_{7}$. 


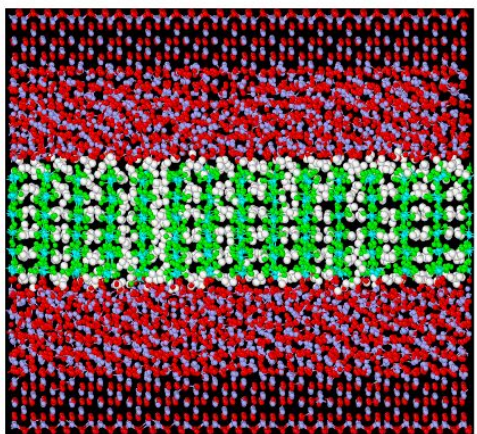

(a) $\mathrm{Na}_{2} \mathrm{SiO}_{3}$-After compression at $250 \mathrm{ps}$

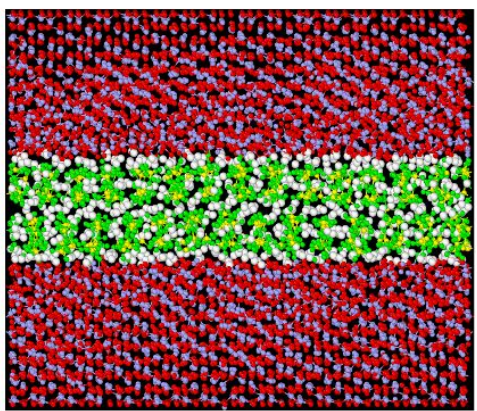

(d) $\mathrm{Na}_{4} \mathrm{P}_{2} \mathrm{O}_{7}$-After compression at 250 ps

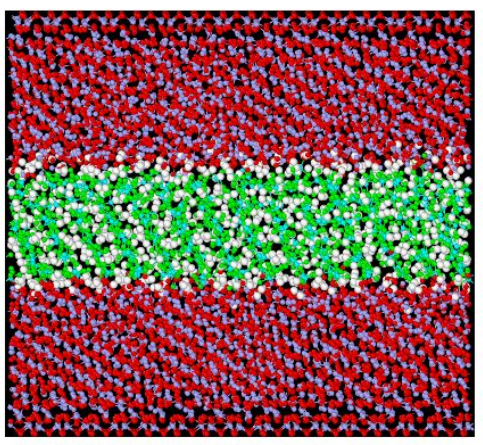

(b) $\mathrm{Na}_{2} \mathrm{SiO}_{3}$-During shear at $375 \mathrm{ps}$

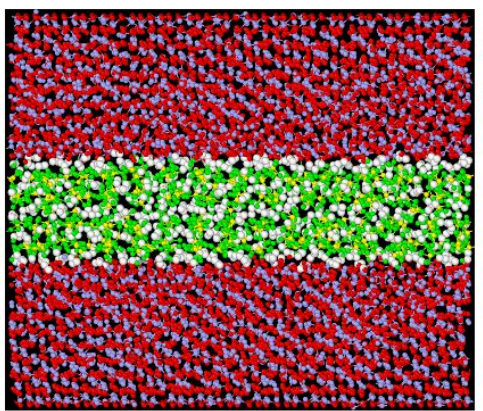

(e) $\mathrm{Na}_{4} \mathrm{P}_{2} \mathrm{O}_{7}$-During shear at $375 \mathrm{ps}$

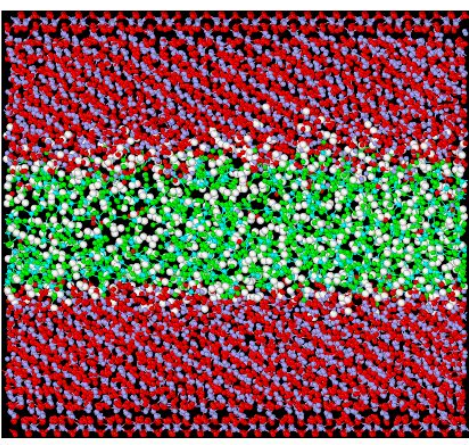

(c) $\mathrm{Na}_{2} \mathrm{SiO}_{3}$-After shear at 1750 ps

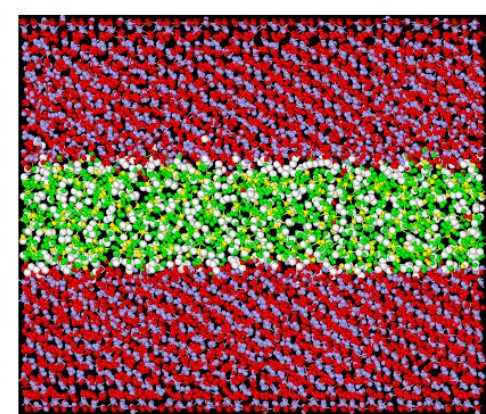

(f) $\mathrm{Na}_{4} \mathrm{P}_{2} \mathrm{O}_{7}$-After shear at $1750 \mathrm{ps}$

Fig. S4 Snapshots of confined-shear systems of sodium pyrophosphate and sodium metasilicate glasses at the beginning (250 ps), transition stage (375 ps) and after shearing process (1750 ps). Fe and $\mathrm{O}$ of surface are respectively purple and red, while the corresponding colors of white, green, aqua, and yellow are for $\mathrm{Na}, \mathrm{O}, \mathrm{Si}$, and $\mathrm{P}$ of lubricants. These settings are applied throughout this work.

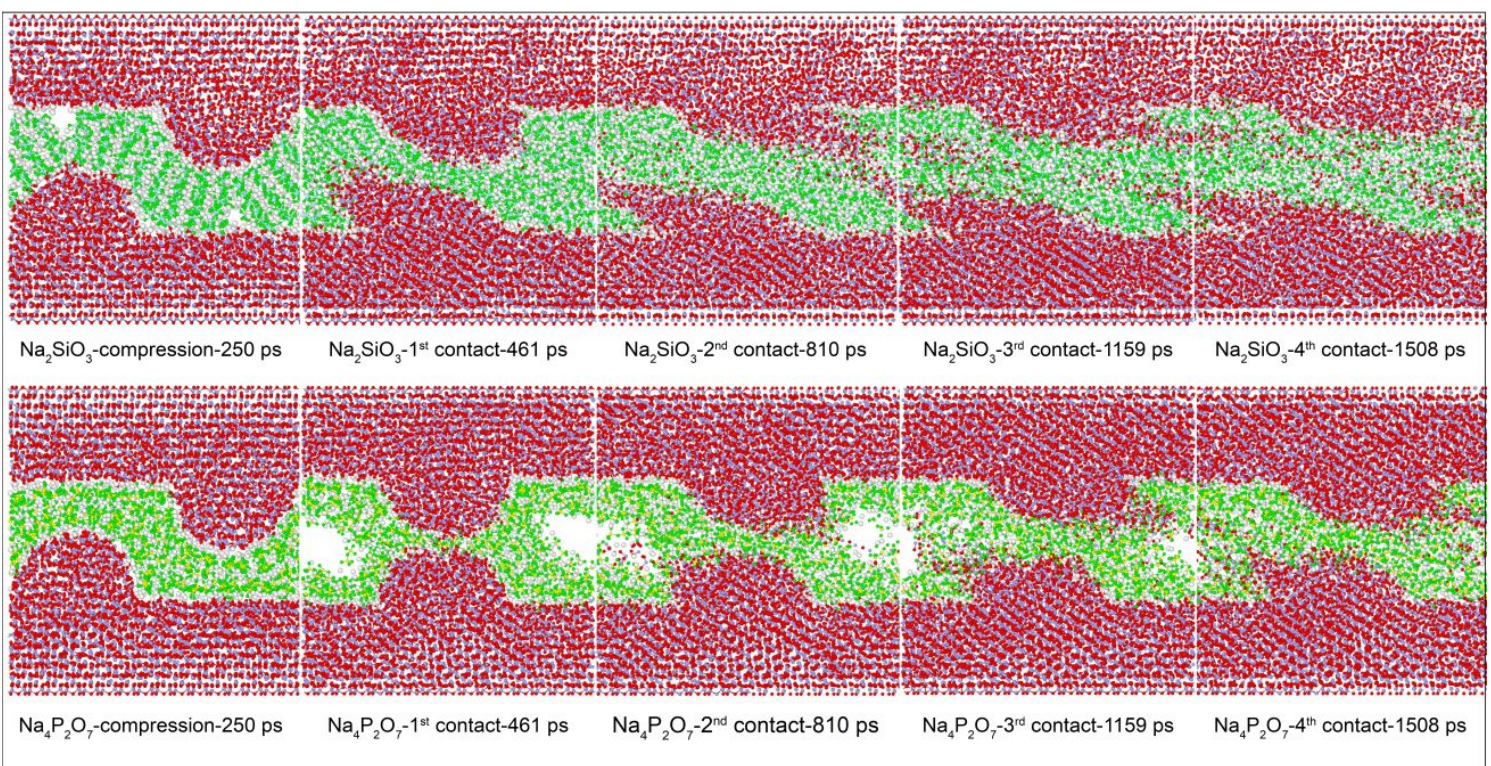

Fig. S5 Snapshots of atomic structure of confined shear systems of sodium pyrophosphate and sodium metasilicate lubricants. 


\section{Appendix A: Equation of State}

The Birch-Murnaghan isothermal equation of state, published in 1947 by Albert Francis Birch of Harvard, ${ }^{1}$ describes the relationship between the volume of a body and the applied pressure. Birch proposed this equation based on the work of Francis Dominic Murnaghan of Johns Hopkins University published in $1944,{ }^{2}$ so that the equation is named in honor of both scientists.

From the third-order Birch-Murnaghan isothermal equation of state is given by

$$
\mathrm{P}(\mathrm{V})=\frac{3 \mathrm{~B}_{0}}{2}\left(\left(\frac{\mathrm{V}_{0}}{\mathrm{~V}}\right)^{\frac{7}{3}}-\left(\frac{\mathrm{V}_{0}}{\mathrm{~V}}\right)^{\frac{5}{3}}\right)\left(1+\frac{3}{4}\left(\mathrm{~B}_{0}^{\prime}-4\right)\left(\left(\frac{\mathrm{V}_{0}}{\mathrm{~V}}\right)^{\frac{2}{3}}-1\right)\right)
$$

where $\mathrm{P}$ is the pressure, $\mathrm{V}_{0}$ is the reference volume, $\mathrm{V}$ is the deformed volume, $\mathrm{B}_{0}$ is the bulk modulus, and $\mathrm{B}_{0}^{\prime}$ is the derivative of the bulk modulus with respect to pressure. The bulk modulus and its derivative are usually obtained from fits to experimental data and are defined as

$$
\mathrm{B}_{0}=-\mathrm{V}\left(\frac{\partial \mathrm{P}}{\partial \mathrm{V}}\right)_{\mathrm{P}=0} \text { and } \mathrm{B}_{0}^{\prime}=\left(\frac{\partial \mathrm{B}}{\partial \mathrm{P}}\right)_{\mathrm{P}=0}
$$

The expression for the equation of state (EoS) is obtained by expanding the free energy $f$ in the form of a series:

$$
\mathrm{f}=\frac{1}{2}\left(\left(\frac{\mathrm{V}}{\mathrm{V}_{0}}\right)^{-\frac{2}{3}}-1\right)
$$

The internal energy, $\mathrm{E}(\mathrm{V})$, is found by integrating equation (1):

$$
E(V)=E_{0}+\frac{9 B_{0} V_{0}}{16}\left\{B_{0}^{\prime}\left[\left(\frac{V_{0}}{V}\right)^{\frac{2}{3}}-1\right]^{3}+\left[\left(\frac{V_{0}}{V}\right)^{\frac{2}{3}}-1\right]^{2}\left[6-4\left(\frac{V_{0}}{V}\right)^{\frac{2}{3}}\right]\right\}
$$

If $E_{0}$ is moved to the left side of equation (4), we will have the term $E(V)-E_{0}$, which is called $d E(V)$. Equation (4) will become:

$$
\mathrm{dE}(\mathrm{V})=\frac{9 \mathrm{~B}_{0} \mathrm{~V}_{0}}{16}\left[\left(\frac{\mathrm{V}_{0}}{\mathrm{~V}}\right)^{\frac{2}{3}}-1\right]\left\{6+\mathrm{B}_{0}^{\prime}\left[\left(\frac{\mathrm{V}_{0}}{\mathrm{~V}}\right)^{\frac{2}{3}}-1\right]-4\left(\frac{\mathrm{V}_{0}}{\mathrm{~V}}\right)^{\frac{2}{3}}\right\}
$$

\section{Appendix B: ReaxFF for $\mathrm{Si} / \mathrm{P} / \mathrm{Na} / \mathrm{Fe} / \mathrm{O}$ systems}

Reactive MD-force field 


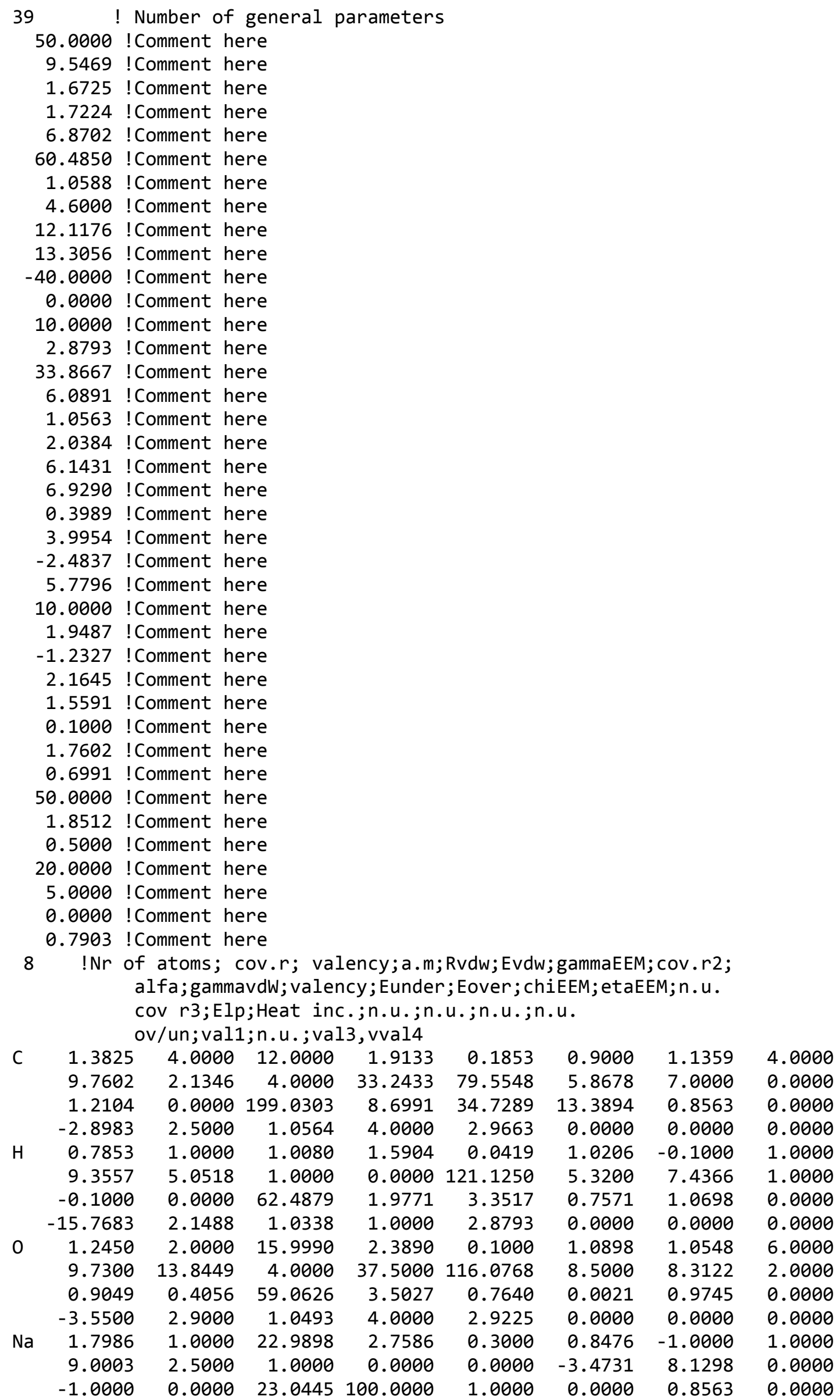




$\begin{array}{rrrrrrrrr} & -4.1479 & 3.9900 & 1.0338 & 8.0000 & 2.5791 & 0.0000 & 0.0000 & 0.0000 \\ \mathrm{P} & 1.5994 & 3.0000 & 30.9738 & 1.5770 & 0.1983 & 0.4655 & 1.3000 & 5.0000 \\ & 10.7864 & 2.7884 & 5.0000 & 0.0000 & 0.0000 & 3.4186 & 5.3855 & 0.0000 \\ & -1.0000 & 3.3786 & 125.6300 & 0.5475 & 11.9674 & 17.3824 & 0.0000 & 0.0000 \\ & -13.7379 & 2.8674 & 1.0338 & 5.0000 & 2.8793 & 0.0000 & 0.0000 & 0.0000 \\ \mathrm{Fe} & 2.0843 & 3.0000 & 55.8450 & 1.8993 & 0.1873 & 0.7264 & -1.0000 & 3.0000 \\ & 11.0534 & 2.2637 & 3.0000 & 0.0000 & 18.3725 & 1.2457 & 7.3021 & 0.0000 \\ & -1.2000 & 0.0000 & 66.4838 & 30.0000 & 1.0000 & 0.0000 & 0.8563 & 0.0000 \\ & -16.2040 & 2.7917 & 1.0338 & 6.0000 & 2.5791 & 0.0000 & 0.0000 & 0.0000 \\ \mathrm{Si} & 2.1932 & 4.0000 & 28.0600 & 1.8951 & 0.1737 & 0.8112 & 1.2962 & 4.0000 \\ & 11.3429 & 5.2054 & 4.0000 & 21.7115 & 139.9309 & 4.0081 & 5.7104 & 0.0000 \\ & -1.0000 & 0.0000 & 128.2031 & 9.0751 & 23.8188 & 0.8381 & 0.8563 & 0.0000 \\ & -4.1684 & 2.0754 & 1.0338 & 4.0000 & 2.5791 & 0.0000 & 0.0000 & 0.0000 \\ \mathrm{~B} & 1.2185 & 3.0000 & 10.8110 & 1.4493 & 0.1853 & 0.9000 & 1.2083 & 3.0000 \\ & 9.7602 & 2.1346 & 3.0000 & 33.2433 & 79.5548 & 6.8775 & 6.7020 & 0.0000 \\ & 1.0593 & 0.0000 & 221.4046 & 8.6991 & 34.7289 & 13.3894 & 0.8563 & 0.0000 \\ & -2.8983 & 2.5000 & 1.0564 & 3.0000 & 2.9663 & 0.0000 & 0.0000 & 0.0000\end{array}$

29 ! Nr of bonds; Edis1;LPpen;n.u.;pbe1;pbo5;13corr;pbo6

pbe2;pbo3;pbo4;n.u.;pbo1;pbo2;ovcorr

\begin{tabular}{|c|c|c|c|c|c|c|c|c|c|}
\hline \multirow[t]{2}{*}{1} & 1 & 156.5953 & 100.0397 & 80.0000 & -0.8157 & -0.4591 & 1.0000 & 37.7369 & 0.4235 \\
\hline & & 0.4527 & -0.1000 & 9.2605 & 1.0000 & -0.0750 & 6.8316 & 1.0000 & 0.0000 \\
\hline \multirow[t]{2}{*}{1} & 2 & 170.2316 & 0.0000 & 0.0000 & -0.5931 & 0.0000 & 1.0000 & 6.0000 & 0.7140 \\
\hline & & 5.2267 & 1.0000 & 0.0000 & 1.0000 & -0.0500 & 6.8315 & 0.0000 & .0000 \\
\hline \multirow[t]{2}{*}{1} & 3 & 160.4802 & 105.1693 & 23.3059 & -0.3873 & -0.1613 & 1.0000 & 10.8851 & 1.0000 \\
\hline & & 0.5341 & -0.3174 & 7.0303 & 1.0000 & -0.1463 & 5.2913 & 0.0000 & 0.0000 \\
\hline \multirow[t]{2}{*}{1} & 5 & 0.0000 & 0.0000 & 0.0000 & 0.2171 & -0.1418 & 1.0000 & 13.1260 & 0.6000 \\
\hline & & 0.3601 & -0.2500 & 20.0000 & 1.0000 & -0.2000 & 10.0000 & 1.0000 & 0.0000 \\
\hline \multirow[t]{2}{*}{1} & 6 & 103.2582 & 0.0000 & 0.0000 & 0.8660 & 0.0000 & 1.0000 & 36.0000 & 0.0100 \\
\hline & & 0.9651 & -0.3500 & 15.0000 & 1.0000 & -0.1399 & 4.3334 & 1.0000 & 0.0000 \\
\hline \multirow[t]{2}{*}{1} & 7 & 108.3910 & 95.0233 & 0.0000 & 0.1129 & -0.5558 & 1.0000 & 17.2117 & 0.4568 \\
\hline & & 0.2424 & -0.2378 & 10.1163 & 1.0000 & -0.1020 & 5.7156 & 1.0000 & 0.0000 \\
\hline \multirow[t]{2}{*}{2} & 2 & 156.0973 & 0.0000 & 0.0000 & -0.1377 & 0.0000 & 1.0000 & 6.0000 & 0.8240 \\
\hline & & 2.9907 & 1.0000 & 0.0000 & 1.0000 & -0.0593 & 4.8358 & 0.0000 & 0.0000 \\
\hline \multirow[t]{2}{*}{2} & 3 & 180.4373 & 0.0000 & 0.0000 & -0.8074 & 0.0000 & 1.0000 & 6.0000 & 0.5514 \\
\hline & & 1.2490 & 1.0000 & 0.0000 & 1.0000 & -0.0657 & 5.0451 & 0.0000 & 0.0000 \\
\hline \multirow[t]{2}{*}{2} & 4 & 0.0000 & 0.0000 & 0.0000 & -1.0000 & -0.3000 & 1.0000 & 36.0000 & 0.7000 \\
\hline & & 10.1151 & -0.3500 & 25.0000 & 1.0000 & -0.1053 & 8.2003 & 1.0000 & 0.0000 \\
\hline \multirow[t]{2}{*}{2} & 5 & 0.0000 & 0.0000 & 0.0000 & 0.2250 & -0.1418 & 1.0000 & 13.1260 & 0.6000 \\
\hline & & 0.3912 & -0.1310 & 0.0000 & 1.0000 & -0.2000 & 10.0000 & 000 & 0.0000 \\
\hline \multirow[t]{2}{*}{2} & 6 & 77.3903 & 0.0000 & 0.0000 & 0.4011 & 0.0000 & 1.0000 & 6.0000 & 0.1228 \\
\hline & & 0.6165 & 1.0000 & 0.6 & 1.0000 & -0.1658 & 5.29 & 00 & 0.0000 \\
\hline \multirow[t]{2}{*}{2} & 7 & 250.0000 & 0.0000 & 0.0000 & -0.7128 & 0.0000 & 1.0000 & 6.0000 & 0.1186 \\
\hline & & 18.5790 & 1.0000 & 0.0000 & 1.0000 & -0.0731 & 7.4983 & 0.0000 & 0.0000 \\
\hline \multirow[t]{2}{*}{2} & 8 & 177.4103 & 0.0000 & 0.0000 & -0.4601 & -0.3000 & 1.0000 & 25.0000 & 0.4971 \\
\hline & & 9.2806 & 0.0000 & 0.0000 & 1.0000 & -0.0722 & 5.1245 & 1.0000 & 0.0000 \\
\hline \multirow[t]{2}{*}{3} & 3 & 142.2858 & 145.0000 & 50.8293 & 0.2506 & -0.1000 & 1.0000 & 29.7503 & 0.6051 \\
\hline & & 0.3451 & -0.1055 & 9.0000 & 1.0000 & -0.1225 & 5.5000 & 1.0000 & 0.0000 \\
\hline \multirow[t]{2}{*}{3} & 4 & 31.0064 & 0.0000 & 0.0000 & 0.1816 & -0.3000 & 1.0000 & 36.0000 & 0.4059 \\
\hline & & 19.1117 & -0.3500 & 25.0000 & 1.0000 & -0.1974 & 6.5386 & 1.0000 & 0.0000 \\
\hline \multirow[t]{2}{*}{3} & 5 & 81.3817 & 119.8068 & 0.0000 & 0.8652 & -0.5000 & 1.0000 & 25.0000 & 0.2000 \\
\hline & & 3.5797 & -0.2067 & 16.0316 & 1.0000 & -0.2491 & 7.9507 & 1.0000 & 0.0000 \\
\hline \multirow[t]{2}{*}{3} & 6 & 75.3857 & 0.0000 & 0.0000 & 0.2110 & -0.4469 & 1.0000 & 6.0000 & 0.0148 \\
\hline & & 1.9760 & 1.0000 & 0.0000 & 1.0000 & -0.9251 & 9.9008 & 1.0000 & 0.0000 \\
\hline \multirow[t]{2}{*}{3} & 7 & 261.9074 & 5.9533 & 0.0000 & -0.6223 & -0.3000 & 1.0000 & 36.0000 & 0.7275 \\
\hline & & 10.1541 & -0.2366 & 29.7817 & 1.0000 & -0.1083 & 8.5924 & 6.0658 & 0.0000 \\
\hline \multirow[t]{2}{*}{3} & 8 & 235.2369 & 114.6973 & 0.0000 & -0.9000 & -0.2500 & 1.0000 & 25.0000 & 0.5534 \\
\hline & & 0.5237 & -0.2746 & 13.4037 & 1.0000 & -0.0898 & 6.0651 & 1.0000 & 0.0000 \\
\hline \multirow[t]{2}{*}{4} & 4 & 104.6461 & 0.0000 & 0.0000 & -0.7273 & 0.3000 & 0.0000 & 25.0000 & 0.1919 \\
\hline & & 6.6441 & -0.4000 & 12.0000 & 1.0000 & -0.0345 & 5.0063 & 0.0000 & 0.0000 \\
\hline
\end{tabular}




$\begin{array}{rrrrrrrrrr}4 & 5 & 0.0000 & 0.0000 & 0.0000 & -0.1228 & 0.2190 & 1.0000 & 35.0000 & 0.8100 \\ & & 3.2849 & 1.0000 & 0.9636 & 1.0000 & -0.9038 & 1.3783 & 1.0000 & 0.0000 \\ 4 & 6 & 0.0000 & 0.0000 & 0.0000 & 0.2500 & -0.5000 & 1.0000 & 35.0000 & 0.6000 \\ & & 0.5000 & -0.5000 & 20.0000 & 1.0000 & -0.0001 & 10.0000 & 1.0000 & 0.0000 \\ 4 & 7 & 0.0000 & 0.0000 & 0.0000 & 0.2500 & -0.5000 & 1.0000 & 35.0000 & 0.6000 \\ & & 0.5000 & -0.5000 & 20.0000 & 1.0000 & -0.2000 & 10.0000 & 1.0000 & 0.0000 \\ 5 & 5 & 77.6075 & 23.0652 & 9.0151 & 0.3170 & -0.5000 & 1.0000 & 35.0000 & 0.0402 \\ & & 13.4229 & -0.4110 & 22.3594 & 1.0000 & -0.0279 & 9.1607 & 1.0000 & 0.0000 \\ 5 & 6 & 0.0000 & 0.0000 & 0.0000 & -0.6998 & -0.5924 & 0.0000 & 36.0000 & 0.2723 \\ & & -0.2496 & -0.6423 & 17.4444 & 1.0000 & -0.0691 & 4.9408 & 0.0000 & 0.0000 \\ 6 & 6 & 27.0485 & 0.0000 & 0.0000 & 0.7994 & -0.2469 & 0.0000 & 16.0000 & 0.1520 \\ & & 1.0000 & -0.2770 & 15.0000 & 1.0000 & -0.0880 & 6.6348 & 0.0000 & 0.0000 \\ 6 & 7 & 0.0000 & 0.0000 & 0.0000 & 0.0000 & -0.5000 & 1.0000 & 35.0000 & 0.6000 \\ & & 0.5000 & -0.5000 & 20.0000 & 1.0000 & -0.2000 & 10.0000 & 1.0000 & 0.0000 \\ 7 & 7 & 70.9120 & 54.0531 & 30.0000 & 0.4931 & -0.3000 & 1.0000 & 16.0000 & 0.0392 \\ & & 0.2476 & -0.8055 & 7.1248 & 1.0000 & -0.1009 & 8.7229 & 0.0000 & 0.0000 \\ 8 & 8 & 189.9665 & 82.7749 & 72.0177 & -0.8157 & -0.4591 & 1.0000 & 37.7369 & 0.4235 \\ & & 0.4527 & -0.1000 & 9.2605 & 1.0000 & -0.0750 & 6.8316 & 1.0000 & 0.0000\end{array}$

20 ! Nr of off-diagonal terms; Ediss;Ro;gamma;rsigma;rpi;rpi2

$\begin{array}{rrrrrrrr}1 & 2 & 0.1219 & 1.4000 & 9.8442 & 1.1203 & -1.0000 & -1.0000 \\ 1 & 3 & 0.1131 & 1.8523 & 9.8442 & 1.2775 & 1.1342 & 1.0621 \\ 1 & 6 & 0.3219 & 1.4560 & 10.9553 & 1.4329 & -1.0000 & -1.0000 \\ 1 & 7 & 0.0541 & 2.0811 & 13.5179 & 1.7778 & 1.5840 & -1.0000 \\ 2 & 3 & 0.0344 & 1.6800 & 10.3247 & 0.9013 & -1.0000 & -1.0000 \\ 2 & 5 & 0.1744 & 1.7715 & 10.4931 & 0.0100 & 0.0100 & -1.0000 \\ 2 & 6 & 0.0208 & 1.8797 & 11.0601 & 1.3942 & -1.0000 & -1.0000 \\ 2 & 7 & 0.2000 & 1.5207 & 12.9535 & 1.2125 & -1.0000 & -1.0000 \\ 2 & 8 & 0.0526 & 1.5010 & 11.2019 & 1.1648 & -1.0000 & -1.0000 \\ 3 & 4 & 0.2000 & 1.3405 & 17.0045 & 1.6861 & -1.0000 & -1.0000 \\ 3 & 5 & 0.0450 & 1.7136 & 11.6409 & 1.7097 & 1.4401 & -1.0000 \\ 3 & 6 & 0.0724 & 1.1776 & 20.0000 & 2.0902 & -1.0000 & -1.0000 \\ 3 & 7 & 0.2000 & 1.9048 & 10.8374 & 1.7163 & 1.2444 & -1.0000 \\ 3 & 8 & 0.0960 & 2.1711 & 10.3073 & 1.3579 & 1.2435 & -1.0000 \\ 4 & 5 & 0.1682 & 1.1937 & 8.1129 & 0.9312 & -1.0000 & -1.0000 \\ 4 & 6 & 0.2053 & 1.5393 & 5.8090 & 1.1111 & -1.0000 & -1.0000 \\ 4 & 7 & 0.1174 & 1.9434 & 17.1734 & -1.0000 & -1.0000 & -1.0000 \\ 5 & 6 & 0.3080 & 1.0117 & 13.6865 & 1.0230 & -1.0000 & -1.0000 \\ 6 & 7 & 0.1811 & 1.8972 & 1.0000 & -1.0000 & -1.0000 & -1.0000 \\ 6 & 8 & 0.3080 & 1.0117 & 13.6865 & -1.0000 & -1.0000 & -1.0000\end{array}$

103 ! Nr of angles;at1;at2;at3;Thetao,o;ka;kb;pv1;pv2

$\begin{array}{rrrrrrrrrr}1 & 1 & 1 & 67.2326 & 22.0695 & 1.6286 & 0.0000 & 1.7959 & 15.4141 & 1.8089 \\ 1 & 1 & 2 & 65.2527 & 14.3185 & 6.2977 & 0.0000 & 0.5645 & 0.0000 & 1.1530 \\ 1 & 1 & 3 & 49.5561 & 7.3771 & 4.9568 & 0.0000 & 0.7533 & 15.9906 & 1.0010 \\ 1 & 1 & 6 & 74.8790 & 30.0000 & 2.0000 & 0.0000 & 2.0334 & 0.0000 & 1.0928 \\ 1 & 1 & 7 & 72.5239 & 22.3583 & 2.0393 & 0.0000 & 1.0031 & 0.0000 & 1.0400 \\ 1 & 2 & 1 & 0.0000 & 3.4110 & 7.7350 & 0.0000 & 0.0000 & 0.0000 & 1.0400 \\ 1 & 2 & 2 & 0.0000 & 0.0000 & 6.0000 & 0.0000 & 0.0000 & 0.0000 & 1.0400 \\ 1 & 2 & 3 & 0.0000 & 25.0000 & 3.0000 & 0.0000 & 1.0000 & 0.0000 & 1.0400 \\ 1 & 2 & 6 & 0.0000 & 5.0000 & 2.0000 & 0.0000 & 1.0000 & 0.0000 & 1.5000 \\ 1 & 2 & 7 & 0.0000 & 2.5000 & 1.0000 & 0.0000 & 1.0000 & 0.0000 & 1.2500 \\ 1 & 3 & 1 & 74.3994 & 44.7500 & 0.7982 & 0.0000 & 3.0000 & 0.0000 & 1.0528 \\ 1 & 3 & 2 & 71.5018 & 21.7062 & 0.4735 & 0.0000 & 0.5186 & 0.0000 & 1.1793 \\ 1 & 3 & 3 & 77.9854 & 36.6201 & 2.0201 & 0.0000 & 0.7434 & 67.0264 & 3.0000 \\ 1 & 3 & 5 & 53.2386 & 27.6683 & 3.5448 & 0.0000 & 0.9129 & 0.0000 & 1.2759 \\ 1 & 3 & 6 & 85.7539 & 12.4507 & 1.7016 & 0.0000 & 0.7773 & 0.0000 & 1.0072 \\ 1 & 3 & 7 & 85.8521 & 12.6881 & 1.0112 & 0.0000 & 1.0000 & 0.0000 & 1.3220 \\ 1 & 5 & 1 & 56.0196 & 40.1896 & 1.0567 & 0.0000 & 0.7180 & 6.2933 & 1.1983 \\ 1 & 5 & 3 & 82.8511 & 35.1702 & 2.2155 & 0.0000 & 1.0000 & 0.0000 & 1.0400 \\ 1 & 6 & 1 & 45.0534 & 43.7115 & 0.1742 & 0.0000 & 1.4685 & 0.0000 & 1.8494\end{array}$




\begin{tabular}{|c|c|c|c|c|c|c|c|c|c|}
\hline & 6 & 2 & 44.1263 & 0.1498 & 0.3272 & 0.0000 & 0.1020 & 0.0000 & \\
\hline 1 & 6 & 6 & 63.3653 & 1.8828 & 8.0000 & 0.0000 & 2.7499 & 0.0000 & 1.0000 \\
\hline & 7 & 1 & 69.1709 & 18.9268 & 2.1226 & 0.0000 & 1.0031 & 0.0000 & .040 \\
\hline 1 & 7 & 2 & 71.8708 & 14.6864 & 2.4702 & 0.0000 & 1.0000 & 0.0000 & 040 \\
\hline & 7 & & 71.7524 & 35.8987 & 1.5000 & 0.0000 & 1.0000 & 0.0000 & 1.048 \\
\hline I & 7 & 7 & 68.9902 & 19.7021 & 2.0587 & 0.0000 & 1.0031 & 0.0000 & 1.040 \\
\hline & 1 & & 70.0840 & 25.3540 & 3.4508 & 0.0000 & 0.0050 & 0.0000 & 3.000 \\
\hline 2 & 1 & 3 & 65.0000 & 14.2057 & 4.8649 & 0.0000 & 0.3504 & 0.0000 & 1.718 \\
\hline & 1 & $\epsilon$ & 2.0180 & 37.8528 & 0.9638 & 0.0000 & 0.1000 & 0.0000 & 1.268 \\
\hline & 1 & 7 & 72.6403 & 13.6964 & 2.4702 & 0.0000 & 1.0000 & 0.0000 & 1.040 \\
\hline & 2 & 2 & 0.0000 & 27.9213 & 5.8635 & 0.0000 & 0.0000 & 0.0000 & 1.040 \\
\hline & 2 & 3 & 0.0000 & 9.7025 & 6.0000 & 0.0000 & 0.0000 & 0.0000 & 1.040 \\
\hline & 2 & 6 & 0.0000 & 0.0100 & 1.0097 & 0.0000 & 2.8060 & 0.0000 & 3.530 \\
\hline & 2 & & 0.0000 & 47.1300 & 6.0000 & 0.0000 & 1.6371 & 0.0000 & 1.040 \\
\hline & 2 & 8 & 0.0000 & 5.0019 & 1.0000 & 0.0000 & 0.0000 & 0.0000 & 1.500 \\
\hline & 3 & 2 & 77.0645 & 10.4737 & 1.2895 & 0.0000 & 0.9924 & 0.0000 & 1.104 \\
\hline & 3 & 3 & 84.9468 & 23.3540 & 1.5057 & 0.0000 & 2.6374 & 0.0000 & 1.302 \\
\hline & 3 & 4 & 79.5256 & 0.0000 & 651 & 0.0000 & 1.1304 & 0.0000 & 1.139 \\
\hline & 3 & 5 & 99.9653 & 44.2516 & 0.1000 & 0.0000 & 3.0722 & 0.0000 & 1.040 \\
\hline & 3 & 6 & 58.9778 & 6.8975 & 755 & 0.0000 & 2323 & 0.0000 & 1.000 \\
\hline & 3 & 7 & 86.7611 & 7.1742 & 1.4013 & 0.0000 & 1.4999 & 0.0000 & 1.040 \\
\hline & 3 & 8 & 72.5977 & 9.2973 & 0. & 0.0000 & 787 & 0.0000 & 3.000 \\
\hline & 5 & 3 & 75.0000 & 25.0000 & 2.0000 & 0.0000 & 1.0000 & 0.0000 & 1.250 \\
\hline & 6 & 2 & 24.6837 & 3.3477 & & 0.0000 & & 0.0000 & 1.764 \\
\hline & 6 & 3 & 31.9418 & 20.2787 & 3.3430 & 0.0000 & 1776 & 0.0000 & 1.669 \\
\hline & 6 & 6 & 32.7903 & 3.9308 & 1.3508 & 0.0000 & 5730 & 0.0000 & 2.417 \\
\hline & 7 & 2 & 75.7983 & 14.4132 & 640 & 0.0000 & 000 & 0.0000 & 1.040 \\
\hline & 7 & 3 & 73.6998 & 40.0000 & 1.8782 & 0.0000 & 4.0000 & 0.0000 & 1.129 \\
\hline & 7 & 7 & 77.2616 & 5.0190 & 944 & 0.0000 & 000 & 0.0000 & 1.040 \\
\hline 2 & 8 & 2 & 62.5987 & 14.6089 & 811 & 0.0000 & 3000 & 0.0000 & 3.000 \\
\hline & 8 & 3 & 65.0000 & 23.9782 & 000 & 0.0000 & & 0.0000 & \\
\hline 2 & 8 & 8 & 55.0000 & 32.2012 & 4.7029 & 0.0000 & 3.0000 & 0.0000 & 1.040 \\
\hline & 1 & 3 & 77.1171 & 39.8746 & 403 & 24.3902 & & -42.97 & 2.124 \\
\hline & 1 & 5 & 50.2929 & 41.6249 & 868 & 0.0000 & 1.0000 & 0.0000 & 1.040 \\
\hline & 1 & 6 & 54.6900 & 12.6123 & 543 & 0.0000 & 2.0000 & 0.0000 & 1.251 \\
\hline 3 & 1 & 7 & 70.0000 & 5.0250 & 000 & 0.0000 & 3000 & 0.0000 & 1.250 \\
\hline & 2 & 3 & 0.0000 & 0.0148 & 6.0000 & 0.0000 & 0.0000 & 0.0000 & 1.040 \\
\hline & 2 & 5 & 0.0000 & 10.0000 & 1.0000 & 0.0000 & 1.0000 & 0.0000 & 1.040 \\
\hline & 2 & 6 & 0.0000 & 0.0100 & 567 & 0.0000 & 0582 & 0000 & 1.351 \\
\hline & 2 & 7 & 0.0000 & 5.0000 & 000 & 0.0000 & 000 & 0000 & 1.250 \\
\hline 3 & 2 & 8 & 0.0000 & 2.5000 & 0.1000 & 0.0000 & 1.6740 & 0.0000 & 2.860 \\
\hline & 3 & 3 & 80.7324 & 30.4554 & 0.9953 & 0.0000 & 1.6310 & 50.0000 & 1.078 \\
\hline & 3 & 4 & 0.0000 & 0.0000 & 000 & 0.0000 & 5743 & 0.0000 & 2.400 \\
\hline & 3 & 5 & 60.0000 & 40.0000 & 4.0000 & 0.0000 & 1.0000 & 0.0000 & 1.040 \\
\hline 3 & 3 & 6 & 62.0924 & 3.5446 & 8.5573 & 0.0000 & 1.9458 & 0.0000 & 2.586 \\
\hline & 3 & 7 & 103.4529 & 26.9589 & 1.3470 & 0.0000 & 1.7728 & 0.0000 & 1.309 \\
\hline 3 & 3 & 8 & 90.0000 & 50.0000 & 1.5928 & 0.0000 & 1.4924 & 0.0000 & 1.040 \\
\hline 3 & 4 & 3 & 8.0033 & 8.1164 & 0.0100 & 0.0000 & 1.7673 & 0.0000 & 4.947 \\
\hline 3 & 4 & 4 & 0.0000 & 0.0000 & 1.0000 & 0.0000 & 1.6743 & 0.0000 & 2.400 \\
\hline 3 & 5 & 3 & 71.7497 & 16.7048 & 3.1929 & -12.5000 & 1.3898 & 0.0000 & 2.967 \\
\hline & 5 & 5 & 70.0000 & 25.0000 & 2.0000 & 0.0000 & 1.0000 & 0.0000 & 1.250 \\
\hline 3 & 5 & 6 & 80.0000 & 40.7321 & 4.7609 & 0.0000 & 0.0100 & 0.0000 & 1.040 \\
\hline & 6 & 3 & 80.5562 & 5.4423 & 1.8307 & 0.0000 & 0.5992 & 0.0000 & 1.423 \\
\hline 3 & 6 & 5 & 80.0000 & 14.6298 & 2.6578 & 0.0000 & 0.5087 & 0.0000 & 2.248 \\
\hline & 6 & 6 & 69.5788 & 6.3351 & 8.3746 & 0.0000 & 1.7643 & 0.0000 & 2.417 \\
\hline 3 & 7 & 3 & 80.1361 & 36.2368 & 0.9504 & 0.0000 & 0.2624 & 0.0000 & 2.078 \\
\hline & 7 & 7 & 90.6812 & 31.1846 & 4.4543 & 0.0000 & 0.5073 & 0.0000 & 2.180 \\
\hline & 8 & 3 & 50.0000 & 25.3190 & 6.0000 & 0.0000 & 3.0000 & 35.0000 & 3.000 \\
\hline & 8 & 8 & 70.0000 & 20.0000 & 4.0000 & 0.0000 & 1.0000 & 0.0000 & 1.250 \\
\hline
\end{tabular}




\begin{tabular}{|c|c|c|c|c|c|c|c|c|c|c|c|c|}
\hline 4 & 3 & 4 & \multicolumn{2}{|c|}{88.7938} & \multicolumn{2}{|r|}{8.3861} & 4.0760 & 0.0000 & 0.0000 & \multicolumn{2}{|c|}{0.0000} & 0.8880 \\
\hline 4 & 3 & 5 & \multicolumn{2}{|c|}{36.4626} & \multicolumn{2}{|r|}{6.7019} & 5.2274 & 0.0000 & 0.2960 & \multicolumn{2}{|c|}{0.0000} & 1.9380 \\
\hline 4 & 3 & 6 & & 0000 & & 0.0000 & 1.0000 & 0.0000 & 1.0000 & & 2000 & 1.0000 \\
\hline 4 & 3 & 7 & & 9867 & & 2.0000 & 5.0000 & 0.0000 & 0.8452 & & 2000 & 1.0000 \\
\hline 4 & 4 & 4 & & 0000 & & 0.0000 & 1.0000 & 0.0000 & 1.6743 & & 000 & 2.4009 \\
\hline 4 & 4 & 5 & & 0000 & & 0.0000 & 1.0000 & 0.0000 & 1.6743 & & 2000 & 2.4009 \\
\hline 4 & 5 & 5 & & 0000 & & 0.0000 & 1.0000 & 0.0000 & 1.6743 & & 000 & 2.4009 \\
\hline 5 & 3 & 5 & & 5590 & & 0.7725 & 9.2578 & -1.9090 & 2.1445 & & 2000 & 1.1963 \\
\hline 5 & 3 & 6 & & 8587 & 108 & 8.4731 & 18.6370 & 0.0000 & 11.7539 & & 2000 & Lo.1359 \\
\hline 5 & 4 & 5 & & 0000 & & 0.0000 & 1.0000 & 0.0000 & 1.6743 & & 2000 & 2.4009 \\
\hline 5 & 5 & 5 & & 0000 & & 7.3367 & 20.0247 & 0.0000 & 4.0137 & & 2000 & 3.0019 \\
\hline 5 & 6 & 6 & & 0000 & & 0.0000 & 1.0000 & 0.0000 & 0.1741 & & 2000 & 1.0400 \\
\hline 6 & 1 & 6 & & 2812 & & 4.6443 & 3.0111 & 0.0000 & 0.1701 & & 2000 & 1.0510 \\
\hline 6 & 2 & 6 & & 0000 & & 0.4428 & 7.9607 & 0.0000 & 2.3717 & & 2000 & 1.1970 \\
\hline 6 & 3 & 6 & & 2224 & & 2.4043 & 4.0388 & 0.0000 & 0.1291 & & 2000 & 0.9714 \\
\hline 6 & 3 & 7 & & 0000 & & 2.4043 & 4.0388 & 0.0000 & 0.1291 & & 2000 & 1.0000 \\
\hline 6 & 3 & 8 & & 0000 & & 2.4043 & 4.0388 & 0.0000 & 0.1291 & & 2000 & 1.0000 \\
\hline 6 & 5 & 6 & & 0000 & & 9.0562 & 4.5151 & 0.0000 & 0.0100 & & 2000 & 1.4639 \\
\hline 7 & 1 & 7 & & 453 & & 7377 & 2.0496 & 0.0000 & 1.0031 & & 2000 & 1.0400 \\
\hline 7 & 2 & 7 & & 0000 & & 4206 & 6.0000 & 0.0000 & 1.6371 & & 2000 & 1.0400 \\
\hline 7 & 3 & 7 & & 450 & & 9739 & 1.7731 & 0.0000 & 3.2548 & & 2000 & 1.0422 \\
\hline 7 & 7 & 7 & & 339 & & 5.4328 & 1.0067 & 0.0000 & 0.1694 & & 2000 & 1.6608 \\
\hline 8 & 2 & 8 & & 0000 & & 000 & 1.0000 & 000 & 1.0000 & & 2000 & 1.0400 \\
\hline 8 & 3 & 8 & & 2683 & & 4.0172 & 6.0000 & 0.0000 & 3.0000 & & 2000 & 1.0400 \\
\hline 8 & 8 & 8 & & 0000 & & 2.0695 & 1.6286 & 0.0000 & 1.7959 & & 4141 & 1.8089 \\
\hline 42 & & ! $\mathrm{N}$ & $\mathrm{o}$ & $=$ tors & sion & ns;at1;at2 & t2; at 3 ; at4; & $4 ; ; \mathrm{V} 1 ; \mathrm{V} 2 ; \mathrm{V}$ & V3;V2(BC & & $n j ; n . u$ & d;n \\
\hline 0 & 1 & 1 & 0 & 0.00 & 300 & 50.0000 & 0.3000 & b -4.0000 & $0 \quad-2.0$ & & 0.0000 & 0.0000 \\
\hline 0 & 1 & 2 & 0 & 0.00 & 300 & 0.0000 & 0.0000 & 0.0000 & 0.0 & & 0.0000 & 0.0000 \\
\hline 0 & 1 & 5 & 0 & 4.00 & 300 & 45.8264 & 0.9000 & -4.0000 & 0.06 & & 0.0000 & 0.0000 \\
\hline 0 & 2 & 2 & 0 & 0. & 00 & 0.0000 & 0.0000 & 0.0000 & 0. & & 0.00 & 0.0000 \\
\hline 0 & 2 & 3 & 0 & 0.00 & 300 & 0.1000 & 0.0200 & -2.5415 & 0.06 & & 0.0000 & 0.0000 \\
\hline 0 & 2 & 7 & 0 & 0.00 & 300 & 0.0000 & 0.1200 & -2.4847 & $0 . e$ & & 0.0000 & 0.0000 \\
\hline 0 & 3 & 3 & 0 & 0.55 & 511 & 25.4150 & 1.1330 & -5.1903 & $-1.0 t$ & & 0.0000 & 0.0000 \\
\hline 0 & 3 & 8 & 0 & -1.95 & 522 & 50.0000 & -0.5000 & -2.5000 & 0.0 & & 0.0000 & 0.0000 \\
\hline 0 & 8 & 8 & 0 & 0.25 & 500 & 50.0000 & 0.3000 & -7.5000 & 0.0 & & 0.0000 & 0.0000 \\
\hline 1 & 1 & 1 & 1 & -0.25 & 0 & 11.5822 & 0.1879 & -4.7057 & -2.2 & & 0.0000 & 0.0000 \\
\hline 1 & 1 & 1 & 2 & -0.25 & 0 & 31.2596 & 0.1709 & -4.6391 & -1.9 & & 0.0000 & 0.0000 \\
\hline 1 & 1 & 1 & 3 & $-0.7 e$ & 8 & 22.2951 & 0.0060 & -2.5000 & -2.1 & & 0.0000 & 0.0000 \\
\hline 1 & 1 & 1 & 5 & -0.32 & 2 & 14.3871 & 0.1823 & -9.8682 & -1.7 & & 0.00 & 0.0000 \\
\hline 1 & 1 & 3 & 1 & 2.00 & 907 & 25.5641 & -0.0608 & -2.6456 & -1.1 & & 0.0000 & 0.0000 \\
\hline 1 & 1 & 3 & 2 & -1.19 & 953 & 42.1545 & -1.0000 & -8.0821 & $-1 . e$ & & 0.0000 & 0.0000 \\
\hline 1 & 1 & 3 & 3 & -0.01 & $L 79$ & 5.0603 & -0.1894 & -2.5000 & -2.03 & & 0.0000 & 0.0000 \\
\hline 1 & 1 & 7 & 3 & -0.57 & 740 & 22.4215 & 0.8787 & -2.7603 & -1.1 & & 0.0000 & 0.0000 \\
\hline 1 & 3 & 3 & 1 & $2.5 e$ & 300 & -0.5332 & 1.0000 & -3.5096 & -2.90 & & 0.0000 & 0.0000 \\
\hline 1 & 3 & 3 & 2 & $-2.5 e$ & 300 & 3.3219 & 0.7180 & -5.2021 & -2.9 & & 0.0000 & 0.0000 \\
\hline 1 & 3 & 3 & 3 & 0.05 & 531 & -17.3983 & 1.0000 & -2.5000 & -2.1 & & 0.0000 & 0.0000 \\
\hline 1 & 3 & 5 & 3 & -0.94 & $\$ 51$ & 8.2456 & 0.5757 & -5.7138 & 0.0 & & 0.0000 & 0.0000 \\
\hline 2 & 1 & 1 & 2 & -0.17 & 770 & 30.0252 & 0.4340 & -5.0019 & -2.06 & & 0.0000 & 0.0000 \\
\hline 2 & 1 & 1 & 3 & -0.35 & 568 & 22.6472 & 0.6045 & -4.0088 & -1.00 & & 0.0000 & 0.0000 \\
\hline 2 & 1 & 3 & 1 & -0.92 & 284 & 34.3952 & 0.7285 & -2.5440 & -2.46 & & 0.0000 & 0.0000 \\
\hline 2 & 1 & 3 & 2 & $-2.5 e$ & 00 & 79.6980 & 1.0000 & -3.5697 & -2.75 & & 0.0000 & 0.0000 \\
\hline 2 & 1 & 3 & 3 & -0.55 & 583 & 80.0000 & 1.0000 & -4.4000 & -3.00 & & 0.0000 & 0.0000 \\
\hline 2 & 1 & 3 & 5 & -0.12 & 220 & 61.5112 & 0.3316 & -5.4970 & 0.00 & & 0.0000 & 0.0000 \\
\hline 2 & 3 & 3 & 2 & 2.25 & 500 & -6.2288 & 1.0000 & -2.6189 & -1.06 & & 0.0000 & 0.0000 \\
\hline 2 & 3 & 3 & 3 & 0.47 & 723 & -12.4144 & -1.0000 & -2.5000 & -1.00 & & 0.0000 & 0.0000 \\
\hline 2 & 3 & 5 & 3 & $-1.5 e$ & 300 & -1.0000 & 0.3045 & -2.5000 & 0.06 & & 0.0000 & 0.0000 \\
\hline 2 & 7 & 7 & 2 & 0.00 & 300 & 0.0000 & 0.0640 & -2.4426 & 0.0 & & 0.0000 & 0.0000 \\
\hline 2 & 7 & 7 & 7 & 0.00 & 300 & 0.0000 & 0.1587 & -2.4426 & 0.00 & & 0.0000 & 0.0000 \\
\hline 3 & 1 & 1 & 3 & -0.05 & 528 & 6.8150 & 0.7498 & -5.0913 & -1.00 & & 0.0000 & 0.0000 \\
\hline
\end{tabular}




\begin{tabular}{lllllllllll}
3 & 1 & 1 & 7 & -0.5740 & 22.4215 & 0.8787 & -2.7603 & -1.1000 & 0.0000 & 0.0000 \\
3 & 1 & 3 & 1 & -2.5000 & 76.0427 & -0.0141 & -3.7586 & -2.9000 & 0.0000 & 0.0000 \\
3 & 1 & 3 & 2 & 0.0345 & 78.9586 & -0.6810 & -4.1777 & -3.0000 & 0.0000 & 0.0000 \\
3 & 1 & 3 & 3 & -2.5000 & 66.3525 & 0.3986 & -3.0293 & -3.0000 & 0.0000 & 0.0000 \\
3 & 3 & 3 & 3 & -2.5000 & -25.0000 & 1.0000 & -2.5000 & -1.0000 & 0.0000 & 0.0000 \\
5 & 1 & 1 & 5 & -0.1452 & 50.0000 & -0.1915 & -8.0773 & -1.7255 & 0.0000 & 0.0000 \\
5 & 3 & 5 & 3 & 0.1946 & 20.0266 & -0.3314 & -8.1095 & 0.0000 & 0.0000 & 0.0000 \\
5 & 5 & 5 & 5 & 3.5590 & 23.6453 & -0.7307 & -4.6741 & 0.0000 & 0.0000 & 0.0000 \\
8 & 3 & 3 & 8 & 2.0000 & 75.0000 & 0.3000 & -5.0000 & 0.0000 & 0.0000 & 0.0000 \\
1 & \multicolumn{1}{c}{$\mathrm{Nr}$ of hydrogen bonds;at1;at2;at3;Rhb;Dehb;vhb1 } & &
\end{tabular}

\section{References}

(1) Birch, F. Finite Elastic Strain of Cubic Crystals. Phys. Rev. 1947, 71 (11), 809-824.

(2) Murnaghan, F. D. The Compressibility of Media under Extreme Pressures. Proceedings of the National Academy of Sciences 1944, 30 (9), 244. 